\title{
Diffraction theory of Fresnel lenses encoded in low-resolution devices
}

\author{
E. Carcolé, J. Campos, and S. Bosch
}

\begin{abstract}
A mathematical model that describes the behavior of low-resolution Fresnel lenses encoded in any low-resolution device (e.g., a spatial light modulator) is developed. The effects of low-resolution codification, such the appearance of new secondary lenses, are studied for a general case. General expressions for the phase of these lenses are developed, showing that each lens behaves as if it were encoded through all pixels of the low-resolution device. Simple expressions for the light distribution in the focal plane and its dependence on the encoded focal length are developed and commented on in detail. For a given codification device an optimum focal length is found for best lens performance. An optimization method for codification of a single lens with a short focal length is proposed.

Key words: Diffraction, Fresnel lenses, zone plates, digital holography, spatial light modulators, array illuminator.
\end{abstract}

\section{Introduction}

During the past few years, spatial light modulators (SLM's) have been used as basic elements in optical setups. SLM's may be useful for displaying images ${ }^{1}$ or filters ${ }^{2}$ in optical processors as well as for use as different types of diffractive optical elements. ${ }^{3,4}$ The possibility of changing the pattern displayed at video frame rates makes these elements useful for real-time applications. Optical pattern recognition by means of correlation is an example of such applications. ${ }^{5}$ In these systems the scene has to be refreshed quickly in order to carry out the recognition process in real time. This process usually needs different filters to achieve recognition, so these have to be changed rapidly in the optical setup. By use of the coding techniques of computer-generated holography, ${ }^{2}$ the required transmissions of correlation filters may be displayed in the SLM. The low dynamic range of the SLM originated the study of binary phase-only filters. ${ }^{6}$ The impulse response of the binary phase-only filter is basically the superposition of two edge-enhanced versions of the original object that can be separated by the addition of a quadratic phase ${ }^{7,8}$ (spherical wave).

The authors are with the Departament de Física Aplicada i Electrònica, Diagonal 647, Barcelona E-08028, Spain.

Received 1 March 1993.

0003-6935/94/020162-13\$06.00/0.

(C) 1994 Optical Society of America.
The codification techniques permit the encoding of complex functions by modulation of only the amplitude or the phase transmission of the SLM. Particularly, spherical waves may be encoded as lowresolution Fresnel-encoded lenses (LRFEL's), with the possibility of modification of their focal length. This feature makes the SLM useful as an imaging system for coherent and incoherent illumination. ${ }^{3}$ The finite resolution worsens lens performance because it becomes impossible to codify the spherical wave exactly.

The aim of this paper is the study of the LRFEL's written in a SLM or any low-resolution device and elaboration of a mathematical model for describing its behavior. The superimposing of the Fresnelencoded lenses' periodicity and the SLM grating causes moiré effects ${ }^{9}$ to appear. In fact, it can be shown that when a LRFEL is encoded, new secondary LRFEL's appear. ${ }^{10}$ With the model proposed the amplitude and intensity of light distribution can be calculated, especially in the focal regions of the LRFEL's. The width and intensity of the maxima in the focal region can be analyzed as a function of the encoded focal length by use of the same mathematical model. The existence of an optimum focal-length lens for a given low-resolution device is demonstrated, and the focal-length range for useful imaging systems is studied.

If the aim is codification of only one lens, the existence of secondary LRFEL's implies an important loss of energy into secondary foci, and the quality of 
the image is reduced. A codification algorithm is proposed for diminishing these effects, concentrating more energy in the principal focus. The final aspect of this lens is justified with the model developed.

\section{Codification of Fresnel Lenses in Low Resolution}

Previous studies report the effects of sampling in the codification of low-resolution Fresnel phase-encoded lenses. ${ }^{10}$ In this section we obtain more general expressions that determine, for a general case, the phase of the secondary lenses that appear because of the finite resolution of the modulators. The resulting expressions are valid for any number of pixels and any focal length.

In general, when a Fresnel lens is to be represented, the transmission function that is to be encoded onto a pixelated device is written as

$$
A(x, y)=L\left[-\frac{K}{2 f}\left(x^{2}+y^{2}\right)+\varphi_{0}\right] \equiv L\left(\varphi+\varphi_{0}\right),
$$

where $f$ is the focal length of the lens, $K=2 \pi / \lambda$, $\varphi$ is the quadratic phase corresponding to a convergent spherical wave, and $\varphi_{0}$ is an arbitrary initial phase. When a binary amplitude lens (zone plate) is encoded, $L\left(\varphi+\varphi_{0}\right)=\operatorname{BIN}\left(\varphi+\varphi_{0}\right)$, where BIN is a binarization function that assigns zero to angles between 0 and $\pi$ and 1 otherwise. If the LRFEL is phase encoded, $L(\varphi)$ is $\exp (i \varphi)$.

We codify this lens in a low-resolution device (for instance, an SLM) that contains $N \times M$ pixels with a center-to-center spacing $(\Delta x, \Delta y)$. The coordinates of pixel centers can be written as

$$
\begin{array}{ll}
x_{n}=[n+1 / 2 P(N)] \Delta x, & n=0,1,-1,2,-2 \ldots, \\
y_{m}=[m+1 / 2 P(M)] \Delta y, & m=0,1,-1,2,-2 \ldots,
\end{array}
$$

where $P(N)$ is a parity function that is 0 for odd $N$ and 1 for even $N$. This function takes into account the use of a coordinate origin at the center of the SLM. In what follows, the coordinates of the center of each pixel are identified by $(n, m)$, the corresponding coefficients of Eq. (2).

When a function (for instance, a comb) is multiplied by a converging spherical wave, $Z(x, y) \equiv$ $\exp \left[-i K\left(x^{2}+y^{2}\right) / 2 f\right]$, the Fourier transform of the initial function, with $\lambda f$ scale, appears at a distance $f$ in the plane at which the wave focuses. ${ }^{11}$ This plane is called the focal plane. The codification always involves a sampling process of the exact Fresnel lens, by means of multiplication with a bidimensional comb function ${ }^{12}$ with spacing $(\Delta x, \Delta y)$. Thus the sampling of the spherical wave to be encoded causes the appearance of an infinity of new foci on the focal plane at points

$$
\begin{aligned}
& x_{k}=k X, \quad X=\frac{\lambda f}{\Delta x}, \quad k=0, \pm 1, \pm 2 \ldots, \\
& y_{l}=l Y, \quad Y=\frac{\lambda f}{\Delta y}, \quad l=0, \pm 1, \pm 2 \ldots
\end{aligned}
$$

Thus in the image plane the encoded lens behaves as an array of lenses centered at coordinates $\left(x_{k}, y_{l}\right)$. Having this in mind, defining $R_{x}$ and $R_{y}$ as

$$
R_{x}=\frac{X}{\Delta x}, \quad R_{y}=\frac{Y}{\Delta y},
$$

and using Eqs. (2-4), we write the sampled version of the Fresnel lens given by Eq. (1) as

$$
\begin{aligned}
& A(n, m) \\
& \quad=L\left(-\pi\left\{\frac{[n+1 / 2 P(N)]^{2}}{R_{x}}+\frac{[m+1 / 2 P(M)]^{2}}{R_{y}}\right\}+\varphi_{0}\right) .
\end{aligned}
$$

$\left(R_{x}, R_{y}\right)$ are the distances in pixels between the foci. By a change in $n$ and $m$, all the pixels in the codification device are considered.

We show that a lens as described by Eq. (5) is being encoded with the origin at each point $\left(x_{k}, y_{l}\right)$ [given by Eq. (3)]. We call $(k, l)$ the order of the lens. By shifting the coordinate origin to this point, we demonstrate that a quadratic phase term (centered at this point) appears. Since the center $\left(x_{k}, y_{l}\right)$ does not usually coincide with the center of a pixel, the new coordinates $(s, t)$ are related to the old ones $(n, m)$ by

$$
\begin{aligned}
& n=k R_{x}+s-D\left(k R_{x}\right), \quad m=l R_{y}+t-D\left(l R_{y}\right), \\
& x_{s}=\left(s-p_{k}\right) \Delta x, \quad y_{t}=\left(t-q_{l}\right) \Delta y, \\
& p_{k}=D\left(k R_{x}\right)-1 / 2 P(N), \quad q_{l}=D\left(l R_{y}\right)-1 / 2 P(M),
\end{aligned}
$$

where $D(x)$ is the fractional part of $x$. Now, $(s, t)$ are integer couples and, together with $\left(x_{s}, y_{t}\right)$, represent coordinates of the centers of pixels. In these expressions, $p_{k}$ and $q_{l}$ take into account the displacement between the center of the $(k, l)$ lens and the centers of pixels. Using Eq. (6) in Eq. (5), we obtain the following result:

$$
\begin{aligned}
A(s, t)= & L\left(\varphi_{0}+\varphi_{1}+\varphi_{2}+\varphi_{3}\right), \\
\varphi_{1}= & -\pi\left(k^{2} R_{x}+l^{2} R_{y}\right)-2 \pi\left\{k\left[1 / 2 P(N)-D\left(k R_{x}\right)\right]\right. \\
& \left.+l\left[1 / 2 P(M)-D\left(l R_{y}\right)\right]\right\} \\
\varphi_{2}= & -2 \pi(k s+l t), \\
\varphi_{3}= & \pi\left[\frac{\left(s-p_{k}\right)^{2}}{R_{x}}+\frac{\left(t-q_{l}\right)^{2}}{R_{y}}\right] .
\end{aligned}
$$

The term $\varphi_{1}$ is a phase shift relative to the phase of the central Fresnel lens. The term $\varphi_{2}$ is always a multiple of $2 \pi$ and therefore has no effect on the codification. The term $\varphi_{3}$ corresponds to the quadratic phase that indicates that a new lens appears, as 
mentioned above. $\varphi_{0}$ affects all lenses. Our result can be written mathematically:

$$
\begin{gathered}
Z(x, y) \sum_{n, m} \delta\left\{x-\left[n+\frac{1}{2} P(N)\right] \Delta x, y-\left[m+\frac{1}{2} P(M)\right] \Delta y\right\} \\
=\exp \left(i \varphi_{1}\right) Z(x-k X, y-l Y) \sum_{s, t} \delta[(x-k X) \\
\left.-\left(s-p_{k}\right) \Delta x,(y-l Y)-\left(t-q_{l}\right) \Delta y\right] .
\end{gathered}
$$

In Fig. 1(a) we see a binary LRFEL, as defined in the second paragraph of this section, for $R_{x}=R_{y}=96$ and $N=M=480$. With these parameters the first term of $\varphi_{1}$ in Eqs. (7) is a multiple of $2 \pi$, and the second term is $-\pi(k+l)$. So an alternating $(0,-\pi)$ phase shift is encoded in the secondary lenses. In Fig. 1(b), $R_{x}=R_{y}=96.5$ and $N=M=480$. For this case the second term always vanishes and the contribution of $\varphi_{1}$ is $(-\pi / 2)\left(l^{2}+k^{2}\right)$. This means that the phase of diagonal lenses is $-\pi\left(l^{2}\right)$ and lenses with alternating $(0,-\pi)$ phases are encoded. For $(k=0, l= \pm 1)$ or $(k= \pm 1, l=0)$ we have lenses with

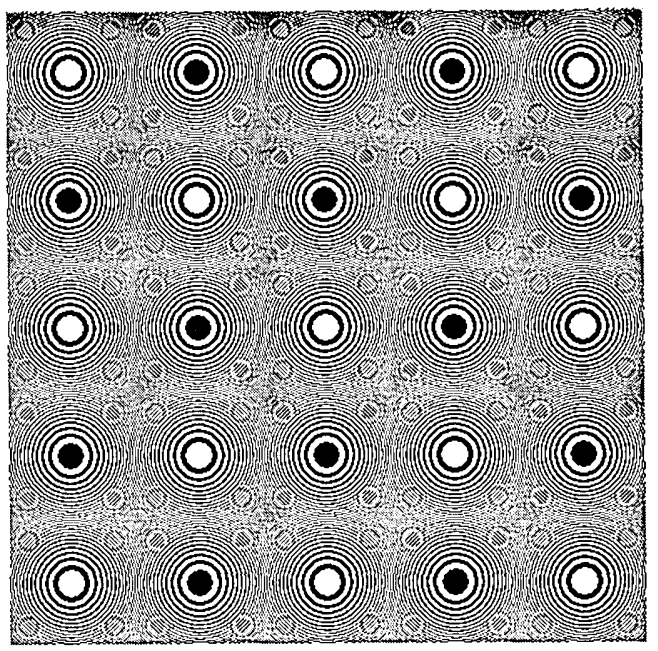

(a)

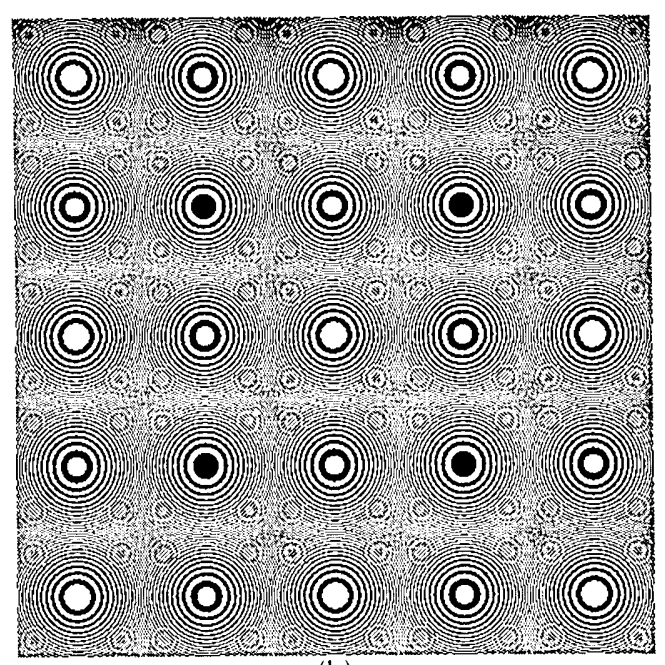

(b)

Fig. 1. Binary LRFEL containing $5 \times 5$ lenses with $N=M=$ 480: (a) $W_{x}=W_{y}=96$, (b) $W_{x}=W_{y}=96.5$. $-\pi / 2$ phase (note the contraction of the first ring in the figure). For $(k= \pm 1, l= \pm 2)$ or $(k= \pm 2, l= \pm 1)$ lenses with $-\pi / 2$ phase appear. For $(k=0, l= \pm 2)$ or $(k= \pm 2, l=0)$ we have 0 phase.

An important conclusion can be deduced from the fact that $(s, t)$ in Eq. (7) can be any of the points of the device for any $(k, l)$ lens: in front of any secondary lens center a spherical wave is encoded throughout all points of the pixelated device. Thus in Section 3 we calculate the amplitude distribution at each focus by using a coordinate system centered on it. This enables us to find simple expressions for the light distribution in the focal plane. Finally, we point out that in Fig. 1 we have not 25 lenses of size $X \times Y$ [distances defined in Eq. (3)] but 25 lenses of $5 X \times 5 Y$, although $X \times Y$ is the apparent size of each lens.

\section{Mathematical Model}

\section{for Low-Resolution-Encoded Lenses}

Let us suppose that we have a pixelated device with lateral dimensions $L_{x} \times L_{y}$ containing $N \times M$ pixels with a center-to-center spacing $(\Delta x, \Delta y)$. Thus $L_{x}=$ $N \Delta x$ and $L_{y}=M \Delta y$. Each pixel is supposed to be a rectangle with dimensions $\left(\Delta x^{\prime}, \Delta y^{\prime}\right)$ and is represented by $\operatorname{rect}\left(x / \Delta x^{\prime}, y / \Delta y^{\prime}\right)$. When a function $L(x, y)$ is encoded in the device, its transmission can be expressed as

$$
\begin{aligned}
A^{\prime}(x, y)= & \left(L ( x , y ) \sum _ { n , m } \delta \left\{x-\left[n+\frac{1}{2} P(N)\right] \Delta x,\right.\right. \\
& \left.\left.y-\left[m+\frac{1}{2} P(M)\right] \Delta y\right\} \operatorname{rect}\left(\frac{x}{L_{x}}, \frac{y}{L_{y}}\right)\right) \\
& * \operatorname{rect}\left(\frac{x}{\Delta x^{\prime}}, \frac{y}{\Delta y^{\prime}}\right),
\end{aligned}
$$

where $*$ stands for convolution. The function $\operatorname{rect}\left(x / L_{x}, y / L_{y}\right)$ defines the pupil according to the finite extension of the codification system.

Let us suppose that we codify a Fresnel phase lens that converges to a distance $f$. Then $L(x, y)$ is $Z(x, y)$. We are interested in the light amplitude distribution $U\left(x_{i}, y_{i}\right)$ in the focal plane from $A^{\prime}(x, y)$. The propagation to the focal plane is represented mathematically by convolution of $A^{\prime}(x, y)$ with a divergent wave: ${ }^{11}$

$$
\begin{aligned}
U\left(x_{i}, y_{i}\right)= & \left(Z ( x , y ) \sum _ { n , m } \delta \left\{x-\left[n+\frac{1}{2} P(N)\right] \Delta x+,\right.\right. \\
& \left.\left.y-\left[m+\frac{1}{2} P(M)\right] \Delta y\right\} \operatorname{rect}\left(\frac{x}{L_{x}}, \frac{y}{L_{y}}\right)\right) \\
& * \operatorname{rect}\left(\frac{x}{\Delta x^{\prime}}, \frac{y}{\Delta y^{\prime}}\right) *\left(\frac{1}{i \lambda f}\right) Z^{-1}(x, y),
\end{aligned}
$$

where $\left(x_{i}, y_{i}\right)$ are the coordinates at the focal plane. The constant phase term $\exp (i K f)$ corresponding to the propagation is omitted. Expression (10) is valid for a plane wave illuminating the lens. We study this case first. A generalization is given in Subsection 3.E. 
As the convolutions associate and commute, Eq. (10) may be written as

$$
\begin{aligned}
U\left(x_{i}, y_{i}\right)= & \left(Z ( x , y ) \sum _ { n , m } \delta \left\{x-\left[n+\frac{1}{2} P(N)\right] \Delta x,\right.\right. \\
& \left.\left.y-\left[m+\frac{1}{2} P(M)\right] \Delta y\right\} \operatorname{rect}\left(\frac{x}{L_{x}}, \frac{y}{L_{y}}\right)\right) \\
& * \operatorname{rect}\left(\frac{1}{i \lambda f}\right) Z^{-1}(x, y) * \operatorname{rect}\left(\frac{x}{\Delta x^{\prime}}, \frac{y}{\Delta y^{\prime}}\right) .
\end{aligned}
$$

This trivial commutation has an important meaning: the amplitude distribution at the focal plane can be calculated as the propagation of an object made of $N \times M$ ideal points and, afterwards, convolved by the function that defines the pixel. This important feature is not dependent on the function being encoded in the device, and it greatly simplifies calculations.

In what follows the amplitude distribution in the foci of the lenses of different orders is presented. The principal focus corresponds to the order $(0,0)$

\section{A. Amplitude Distribution in the $(0,0)$ Focal Region}

To calculate this amplitude, let us compute the first convolution in Eq. (11). Quadratic terms vanish, and only the linear term remains inside the integral. Then, the convolution can be written as a Fourier transform (FT), and Eq. (11) becomes

$$
\begin{aligned}
U_{0,0}\left(x_{i}, y_{i}\right)= & {\left[\frac { Z ^ { - 1 } ( x , y ) } { i \lambda f } \mathscr { F } _ { \lambda f } \left(\sum _ { n , m } \delta \left\{x-\left[n+\frac{1}{2} P(N)\right] \Delta x\right.\right.\right.} \\
& \left.\left.\left.y-\left[m+\frac{1}{2} P(M)\right] \Delta y\right\} \operatorname{rect}\left(\frac{x}{L_{x}}, \frac{y}{L_{y}}\right)\right)\right] \\
& * \operatorname{rect}\left(\frac{x}{\Delta x^{\prime}}, \frac{y}{\Delta y^{\prime}}\right)
\end{aligned}
$$

where $\mathscr{F}_{\lambda f}$ indicates a Fourier transform with $\lambda f$ scale. This FT is easily calculated, and for the focal region we have

$$
\begin{aligned}
U_{0,0}\left(x_{i}, y_{i}\right)= & \left(\frac{L_{x} L_{y}}{\Delta x \Delta y}\right)\left\{\left[\frac{Z^{-1}(x, y)}{i \lambda f}\right]\right. \\
& \times \sum_{n, m} \exp \{-i \pi[n P(N)+m P(M)]\} \\
& \left.\times \operatorname{sinc}\left[\frac{L_{x}(x-n X)}{\lambda f}, \frac{L_{y}(y-m Y)}{\lambda f}\right]\right\} \\
& * \operatorname{rect}\left(\frac{x}{\Delta x^{\prime}}, \frac{y}{\Delta y^{\prime}}\right),
\end{aligned}
$$

where $(X, Y)$ is the distance between two secondary lenses, given by Eq. (3). The two-dimensional sinc is, of course, the product of two one-dimensional ones. If $X$ and $Y$ are much greater than the sinc width $\left(\lambda f / L_{x}, \lambda f / L_{y}\right)$, only the sinc function corresponding to $n=0$ and $m=0$ contributes significantly to the center. Taking into account the values of $X$ and $Y$, we see that this condition is equivalent to $N \gg$ 1 and $M \gg 1$, where $N$ and $M$ are the numbers of pixels in a row and in a column, respectively, of the device. Thus expression (13) can be very well approximated by

$$
\begin{aligned}
U_{0,0}\left(x_{i}, y_{i}\right)= & \frac{1}{i \lambda f}\left(\frac{L_{x} L_{y}}{\Delta x \Delta y}\right)\left\{\exp \left[\frac{i K}{2 f}\left(x^{2}+y^{2}\right)\right]\right. \\
& \left.\times \operatorname{sinc}\left(\frac{L_{x} x}{\lambda f}, \frac{L_{y} y}{\lambda f}\right)\right\} * \operatorname{rect}\left(\frac{x}{\Delta x^{\prime}}, \frac{y}{\Delta y}\right),
\end{aligned}
$$

where $Z^{-1}(x, y)$ is made explicit. This justifies our notation $U_{0,0}\left(x_{i}, y_{i}\right)$.

Equation (14) can also be approximated. We now show that the complex exponential is not important for the calculation in the region in which an appreciable amount of light impinges. The importance of the complex exponential in Eq. (14) arises from the convolution with the rect. Thus the phase variation between opposite vertices of the rect function must be much smaller than $2 \pi$ if the complex exponential is to be neglected:

$$
\begin{aligned}
\frac{K}{2 f}\left[\left(x_{i}+\right.\right. & \left.\left.\frac{\Delta x^{\prime}}{2}\right)^{2}+\left(y_{i}+\frac{\Delta y^{\prime}}{2}\right)^{2}\right] \\
& -\frac{K}{2 f}\left[\left(x_{i}-\frac{\Delta x^{\prime}}{2}\right)^{2}+\left(y_{i}-\frac{\Delta y^{\prime}}{2}\right)^{2}\right] \ll 2 \pi .
\end{aligned}
$$

Using inequality (15) for $\left(x_{i}=X / 2, y_{i}=0\right)$ or $\left(x_{i}=0\right.$, $\left.y_{i}=Y / 2\right)$, we obtain only a $\pi\left(\Delta x^{\prime} / \Delta x\right)$ phase shift caused by, at most, a single half-oscillation of the complex exponential term inside the rect. These values of $x_{i}$ and $y_{i}$ are the sides of a region of size equivalent to the apparent area of a lens, given by $X \times$ $Y$. Operating, and using the parameters defined, we can transform inequality (15) to obtain

$$
\frac{1}{2}\left(\frac{\Delta x^{\prime}}{\Delta x} \frac{x_{i}}{X}+\frac{\Delta y^{\prime}}{\Delta y} \frac{y_{i}}{Y}\right) \ll 1 .
$$

This inequality defines the region in which the complex exponential term can be neglected for lightintensity distribution calculations. This region is called the focal region. Taking as $\left(x_{i}, y_{i}\right)$ the points representing the sinc width $\left(\lambda f / L_{x}, \lambda f / L_{y}\right)$ and the rect width $\left(\Delta x^{\prime}, \Delta y^{\prime}\right)$, inequality $(16)$ is verified for each case if

$$
\begin{aligned}
\frac{1}{2}\left(\frac{\Delta x^{\prime}}{\Delta x} \frac{1}{N}+\frac{\Delta y^{\prime}}{\Delta y} \frac{1}{M}\right) & \ll 1, \\
\frac{1}{2}\left[\left(\frac{\Delta x^{\prime}}{\Delta x}\right)^{2} \frac{1}{R_{x}}+\left(\frac{\Delta y^{\prime}}{\Delta y}\right)^{2} \frac{1}{R_{y}}\right] & \ll 1 .
\end{aligned}
$$

$N \times M$ is the size in pixels of the device, and $R_{x} \times R_{y}$ is the apparent size of the lens measured in pixels; thus the inequalities are always verified for unusual cases. Of course the conditions for the validity of the Fresnel diffraction formulae are also always assumed in this 
study. Then, we can finally write the light distribution amplitude as

$$
U_{0,0}\left(x_{i}, y_{i}\right)=\frac{1}{i \lambda f}\left(\frac{L_{x} L_{y}}{\Delta x \Delta y}\right) \operatorname{sinc}\left(\frac{L_{x} x}{\lambda f}, \frac{L_{y} y}{\lambda f}\right) * \operatorname{rect}\left(\frac{x}{\Delta x^{\prime}}, \frac{y}{\Delta y^{\prime}}\right) \text {. }
$$

Thus in the $(0,0)$ focal region, Eq. (18) is the convolution of a sinc (because of the rectangular pupil) with the rect that defines the shape of any pixel. The actual aspect of this distribution is commented on later on in this paper. If the shapes of the pupil and the pixel are represented by other functions, the only modifications in Eq. (18) are for changing the sinc by the FT of the pupil function and the rect by the function that defines the pixel. This gives general validity to Eq. (18).

\section{B. Amplitude Distribution in a $(k, l)$ Focal Region}

To evaluate this amplitude distribution, we perform a coordinate shift to the center of the $(k, l)$ lens. In Section 2 we demonstrated that, in front of a secondary lens of order $(k, l)$, a spherical wave centered at $(k X, l Y)$ emerges from the object [Eq. (8)]. The only differences with the $(0,0)$ lens are a different phase, given by $\varphi_{1}$ in Eq. (7), and the limitation by a rectangular pupil displaced $(k X, l Y)$ from the new coordinate origin. This last fact is very important; it causes the differences between the $(k, l)$ fócus and the $(0,0)$ focus. Thus in the $(k, l)$ focal region we have

$$
\begin{aligned}
U_{k, l}\left(x_{i}, y_{i}\right)= & \left\{Z ( x , y ) \sum _ { s , t } \delta \left[x-\left(s-p_{k}\right) \Delta x,\right.\right. \\
& \left.\left.y-\left(t-q_{l}\right) \Delta y\right] \operatorname{rect}\left(\frac{x+k X}{L_{x}}, \frac{y+l Y}{L_{y}}\right)\right\} \\
& *\left(\frac{1}{i \lambda f}\right) Z^{-1}(x, y) * \operatorname{rect}\left(\frac{x}{\Delta x^{\prime}}, \frac{y}{\Delta y^{\prime}}\right),
\end{aligned}
$$

where $x_{i}$ and $y_{i}$ are coordinates relative to the $(k, l)$ focus and the phase $\varphi_{1}$ is omitted for simplicity. Taking into account the same approximations as in the preceding case, we can write the corresponding amplitude distribution as

$$
\begin{aligned}
\left.{ }^{-} U_{k, l}\right)\left(x_{i}, y_{i}\right)= & \left(\frac{1}{i \lambda f} \frac{L_{x} L_{y}}{\Delta x \Delta y}\right) \\
& -\left\{\exp \left[i \frac{2 \pi}{\lambda f}(x k X+y l Y)\right] \operatorname{sinc}\left(\frac{L_{x} x}{\lambda f}, \frac{L_{y} y}{\lambda f}\right)\right\} \\
& * \operatorname{rect}\left(\frac{x}{\Delta x^{\prime}}, \frac{y}{\Delta y^{\prime}}\right) .
\end{aligned}
$$

Thus the amplitude distribution at the $(k, l)$ focal region includes a complex oscillatory function whose frequency increases with the distance to the origin, i.e., with $(k X, l Y)$. Besides, it is modulated by a sinc and is then convolved by the pixel shape (a rectangle in our case). When $k=0$ and $l=0$, Eq. (18) is obtained.

For a better understanding of these expressions, let us define the following adimensional constants:

$$
\begin{aligned}
W_{x} & =\frac{L_{x}}{X}, \quad W_{y}=\frac{L_{y}}{Y}{ }^{5} \\
c_{x} & =\frac{\Delta x^{\prime}}{\Delta x}, \quad c_{y}=\frac{\Delta y^{\prime}}{\Delta y},
\end{aligned}
$$

where $W_{x}$ and $W_{y}$ represent the number of lenses in the $x$ and $y$ directions and $c_{x}$ and $c_{y}$ are the ratios of the pixel width to the pixel separation. Let us perform also the following transformation:

$$
\begin{aligned}
u=W_{x}\left(\frac{x}{\Delta x}\right), & v=W_{y}\left(\frac{y}{\Delta y}\right), \\
u_{i}=W_{x}\left(\frac{x_{i}}{\Delta x}\right), & v_{i}=W_{y}\left(\frac{y_{i}}{\Delta y}\right) .
\end{aligned}
$$

Using Eqs. (21) and (22) in Eq. (20) and writing the convolution, we can write the resulting light distribution in a $(k, l)$ focal zone as

$$
\begin{aligned}
U_{k, l}\left(u_{i}, v_{i}\right)= & \left(\frac{\lambda f}{i \Delta x \Delta y}\right) \int_{-a+u_{i}}^{a+u_{i}} \int_{-b+v_{i}}^{b+v_{i}} \\
& \times \exp \left[i 2 \pi\left(\frac{k}{W_{x}} u+\frac{l}{W_{y}} v\right)\right] \operatorname{sinc}(u, v) \mathrm{d} u \mathrm{~d} v \\
a= & \frac{W_{x} c_{x}}{2}, \quad b=\frac{W_{y} c_{y}}{2} .
\end{aligned}
$$

This integral can be factorized in $u$ and $v$. For a given $(k, l)$ order, the integral depends on only four parameters: $W_{x}, W_{y}, c_{x}$, and $c_{y}$. From Eq. (22), $\Delta x$ and $\Delta y$ work as scale factors.

\section{Preliminary Results}

Let us now analyze this integral when the LRFEL is an apparent array of lenses, as in Fig. 1. From Eq. (21), recall that $W_{x}$ is the number of lenses appearing in the $x$ direction (all the comments also apply to the $y$ direction). Particularly, $W_{x}$ is an odd integer when the lenses fit exactly into the $x$ size of the device.

The calculation for $x_{i}=y_{i}=0$ implies that the integral of Eq. (23) is extended to an interval that contains or is contained in the interval $(-1,1)$, that is, the-width of the central lobe of the sinc. Outside of this interval the values of the sinc function decreases quickly, and their contribution to the integral is very small. If $W_{x}$ is an odd integer, the lens of highest order that is encoded in this array is $k_{h}=$ $\left(W_{x}-1\right) / 2$. The quotient $k_{h} / W_{x}$ in the exponential term of Eq. (23) is $1 / 2-1 /\left(2 W_{x}\right) \simeq 1 / 2$; therefore the exponential term contained in the principal maximum of the sinc takes a phase variation of approximately $2 \pi$. This does not imply a large decrease of the integral, because the sinc is not a constant function. If the amplitude is calculated in higher 
orders (that are not encoded in the diffracting device), the phase variation is greater than $2 \pi$, and consequently the amplitude decreases very quickly. This can also be deduced from Eq. (23). The amplitude for any $(k, l)$ focal region at the point $x_{i}=y_{i}=0$ can be written as

$$
U_{k, l}(0,0) \propto \mathscr{F}_{W_{x}, W_{y}}{ }^{-1}\left[\operatorname{sinc}(u, v) \operatorname{rect}\left(\frac{u}{W_{x} c_{x}}, \frac{v}{W_{y} c_{y}}\right)\right],
$$

which is

$$
U_{k, l}(0,0) \propto \operatorname{rect}\left(\frac{k}{W_{x}}, \frac{l}{W_{y}}\right) * \operatorname{sinc}\left(k c_{x}, l c_{y}\right) .
$$

When the conditions $W_{x} \gg 1 / c_{x}$ and $W_{y} \gg 1 / c_{y}$ are fulfilled, we can approximate relation (25) as

$$
U_{k, l}(0,0) \approx \operatorname{rect}\left(\frac{k}{W_{x}}, \frac{l}{W_{y}}\right) .
$$

Then, when a high number of lenses are encoded, they all have approximately the same intensity in the corresponding focus, and the higher orders $\left(k>W_{x} / 2\right.$, $\left.l>W_{y} / 2\right)$ not encoded in the device have a very low intensity. Experimental confirmation of this result has been reported in Ref. (10).

Figure 2 shows the intensity distribution in the focal regions of orders $(0,0),(1,0),(2,0),(2,2)$, and $(3,0)$ for a $5 \times 5$ array of lenses, as in Fig. 1 , computed with Eq. (23). All drawings are represented at the same scale. The conditions taken are $W_{x}=W_{y}=5$, $c_{x}=c_{y}=1$, and $\Delta x=\Delta y$. An experimental configuration compatible with these values is the following: $\lambda=632.8 \mathrm{~nm}, f=1 \mathrm{~m}, N=M=480$, and $\Delta x=\Delta y=$ $0.0812 \mathrm{~mm}$. The intensity differences for orders $(0,0),(0,1),(0,2)$ (which correspond to the principal and two secondary lenses actually encoded) are small but noticeable: the $(2,2)$ order is $\sim 80 \%$ of the $(0,0)$ order. The intensities of the orders not encoded [such as $(0,3)]$ are much smaller.

In the opposite case, from Eq. (25) when $W_{x} \ll 1 / c_{x}$ and $W_{y} \ll 1 / c_{y}$, i.e., a single lens is encoded or we have small pixels, can we write

$$
U_{k, l}(0,0) \propto \sin c\left(k c_{x}, l c_{y}\right)
$$

In this case the light distribution at the center of each focus is modulated by a global sinc. This also implies that for $c_{x}=c_{y}=1$ the intensity at the center of the secondary foci tends to zero.

\section{Asymptotic Limits}

Now we study the asymptotic behavior of the previous expressions as the focal distance $f \rightarrow 0$ or $f \rightarrow \infty$. This can be easily done from Eq. (20). We refer to the $x$ coordinate, but the comments are also valid for $y$.

When $\lambda f / L_{x}$ is much greater than $\Delta x^{\prime}$, i.e., $W_{x} c_{x} \ll$ 1 , the sinc function in Eq. (20) is very wide. Then, for the $(0,0)$ order the convolution of the sinc with the rect has almost no effect on the shape of the sinc, and the amplitude distribution in this order can be written

$$
U_{0,0}\left(x_{i}, y_{i}\right) \simeq \frac{c_{x} c_{y} L_{x} L_{y}}{i \lambda f} \operatorname{sinc}\left(\frac{W_{x} x_{i}}{\Delta x}, \frac{W_{y} y_{i}}{\Delta y}\right) .
$$

For an order other than $(0,0)$ the main lobe of the sinc contains several oscillations of the complex exponential term, making the integral smaller.

If we consider just the opposite case, $\lambda f / L_{x}$ becomes smaller than $\Delta x^{\prime}$, i.e., $W_{x} c_{x} \gg 1$, and the sinc function becomes very narrow (tends to a delta function), never containing even a single oscillation of the complex exponential. In this last case the convolution with the sinc function does not alter the shape of the pixel (in our case the rectangle). Then, for $f \rightarrow 0$ (with many apparent lenses being encoded), Eq. (20) becomes

$$
U_{k, l}\left(x_{i}, y_{i}\right) \propto \frac{1}{i} \frac{\lambda f}{\Delta x \Delta y} \operatorname{rect}\left(\frac{x}{\Delta x^{\prime}}, \frac{y}{\Delta y^{\prime}}\right) .
$$

In conclusion, for a long focal length or for a small number of pixels the limiting shape for the light distribution is the Fourier transform of the pupil function (in our case a sinc). For a short focal length or a high number of pixels the limiting shape for the distribution is that given by the pixel (in our case a small rectangle).

This is illustrated in Fig. 3, in which we show the intensity distributions for $c_{x}=c_{y}=1$ and $W_{x}=W_{y}=$ 0.5 or $W_{x}=W_{y}=13.3$, computed with Eq. (23). We may notice the asymptotic behavior although the values of $W_{x} c_{x}$ and $W_{y} c_{y}$ are close to one. These figures are not normalized.

\section{E. Generalization for Illumination with a Spherical Wave}

Throughout Section 3 we have studied the focusing properties of a LRFEL when illuminated with a plane wave. If a spherical wave with origin at a distance $s$ from the codification plane illuminates the LRFEL, light focuses at another distance, say $s^{\prime}$. Now, the amplitude in this focal plane can be written as

$$
\begin{aligned}
U\left(x_{i}, y_{i}\right)= & \frac{1}{i \lambda f} \int_{-\infty}^{\infty} \int_{-\infty}^{\infty} A^{\prime}(x, y) \exp \left[i\left(\frac{K}{2 s}\right)\left(x^{2}+y^{2}\right)\right] \\
& \times \exp \left\{i\left(\frac{K}{2 s^{\prime}}\right)\left[\left(x_{i}-x\right)^{2}+\left(y_{i}-y\right)^{2}\right]\right\} \mathrm{d} x \mathrm{~d} y,
\end{aligned}
$$

where the convolution is explicitly written. $A^{\prime}(x, y)$ is given by Eq. (9), and $s$ is the distance from the origin of the illuminating wave to the plane of the LRFEL. Taking $s^{\prime}$ from $s^{-1}+s^{\prime-1}=f^{-1}$, we can write Eq. (30) as

$$
\begin{aligned}
U\left(x_{i}, y_{i}\right)= & \frac{1}{i \lambda f} \exp \left[\frac{i K}{2 f \Gamma}\left(1-\frac{1}{\Gamma}\right)\left(x_{i}^{2}+y_{i}^{2}\right)\right] \\
& \times \int_{-\infty}^{\infty} \int_{-\infty}^{\infty} A^{\prime}(x, y) \exp \left[i\left(\frac{K}{2 f}\right)\left(\frac{x_{i}}{\Gamma}-x\right)^{2}\right. \\
& \left.+\left(\frac{y_{i}}{\Gamma}-y\right)^{2}\right] \mathrm{d} x \mathrm{~d} y, \quad \Gamma=\frac{s^{\prime}}{f} .
\end{aligned}
$$




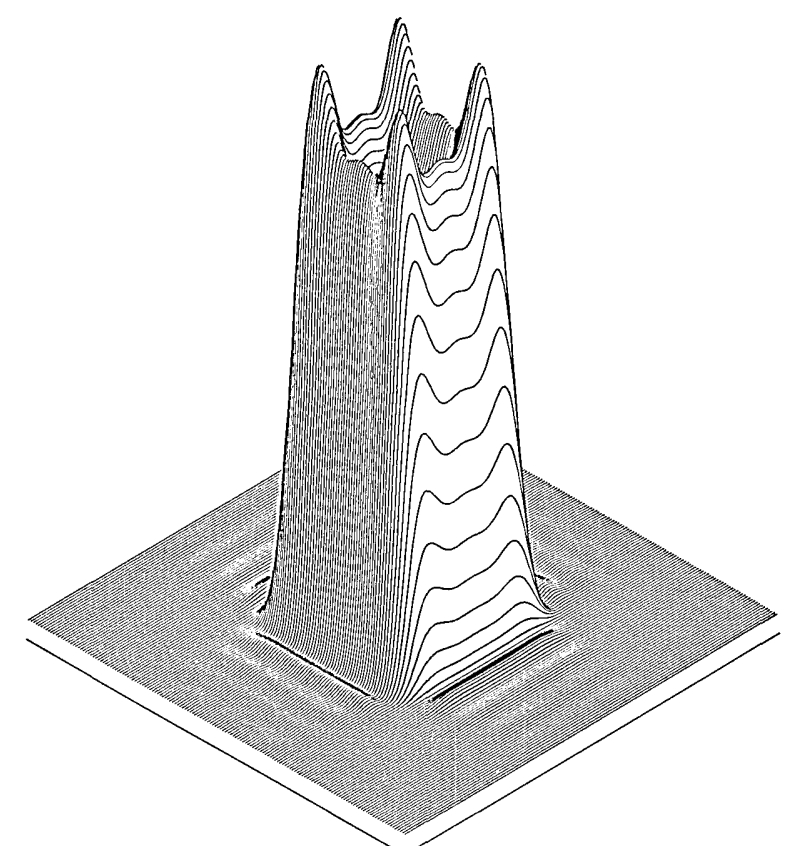

(a)

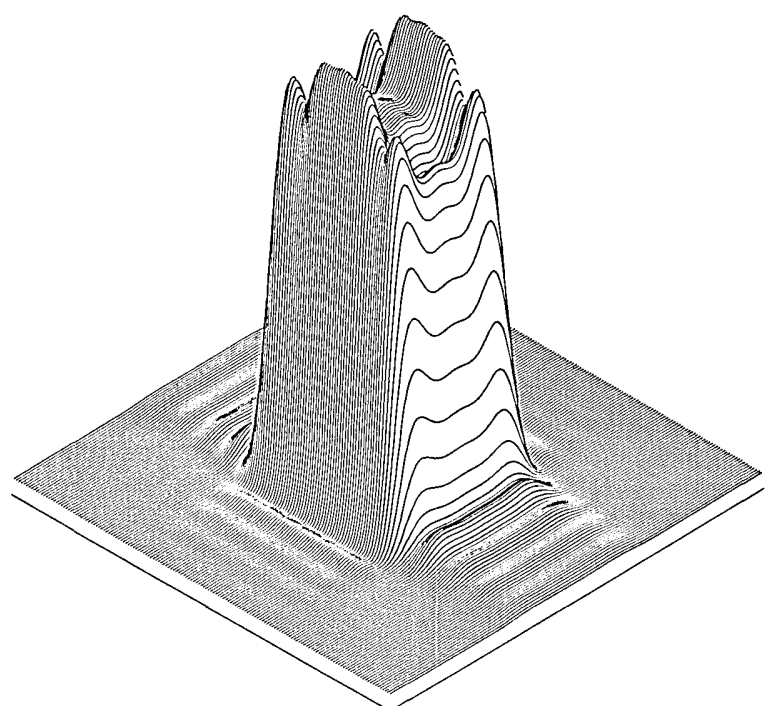

(c)

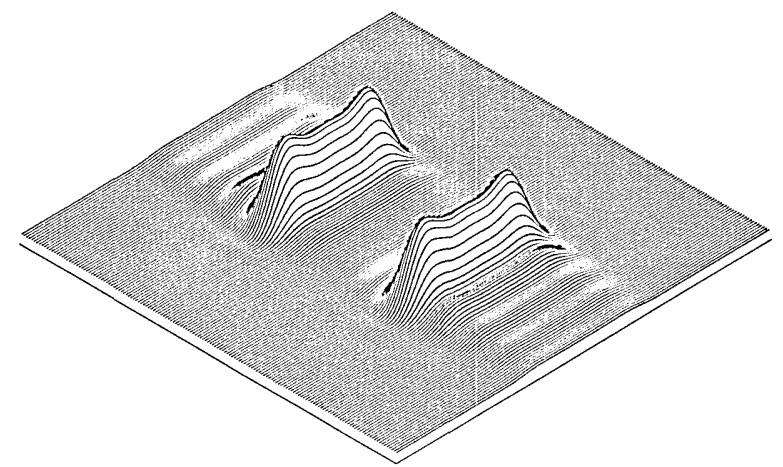

(e)

Thus we have exactly the same light distribution at $s^{\prime}$ as we had before at $f$, i.e., a convolution between the object and the wave converging at a distance $f$ but affected by a factor scale given by $\Gamma$. The complex

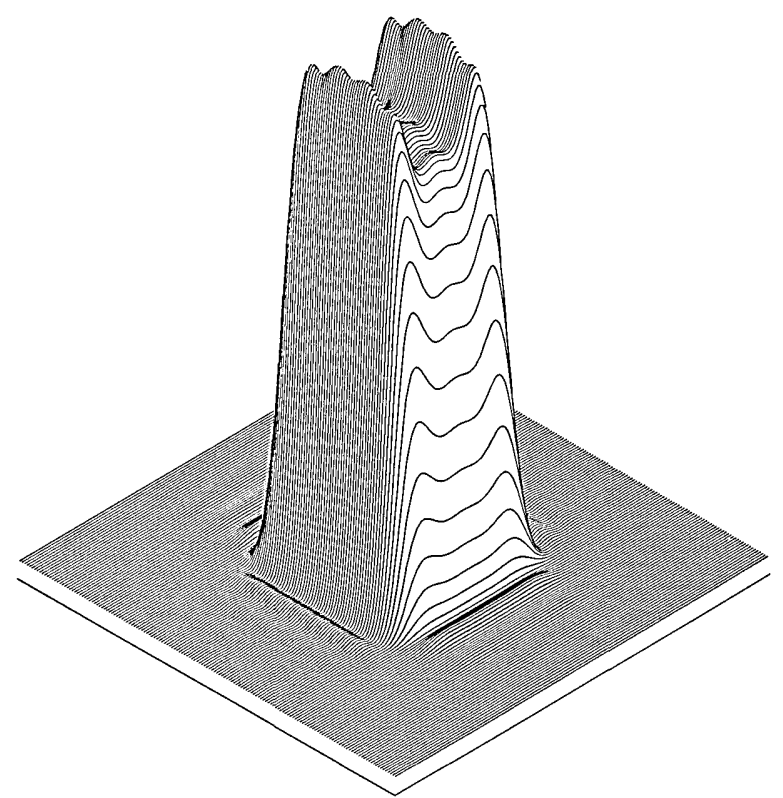

(b)

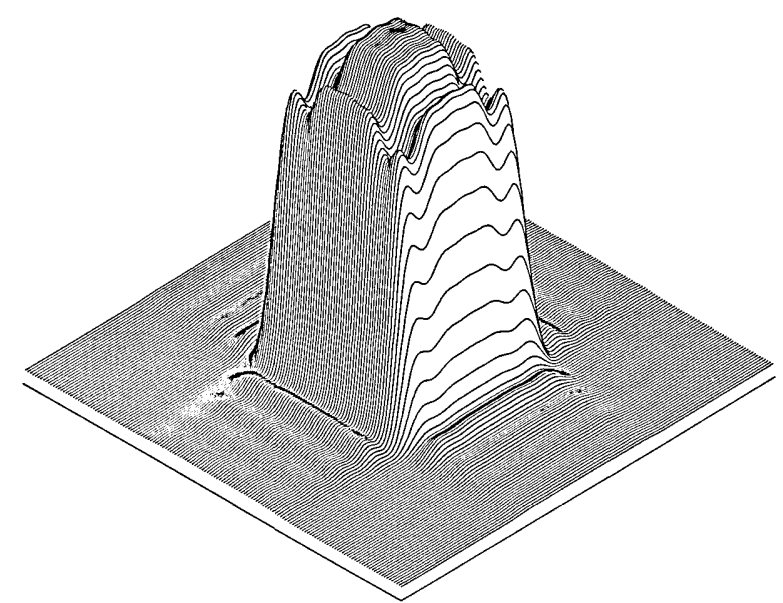

(d)

Fig. 2. Three-dimensional representation of light-intensity distributions in the focal plane for $W_{x}=W_{y}=5, c_{x}=c_{y}=1$, and $\Delta x=$ $\Delta y$ : (a) order $(0,0),(\mathrm{b}) \operatorname{order}(1,0),(\mathrm{c}) \operatorname{order}(2,0),(\mathrm{d}) \operatorname{order}(2,2)$, (e) $\operatorname{order}(3,0)$.

term outside the integral does not affect the intensity. Equation (31) ensures that all our previous treatment is completely general. In the following we suppose plane-wave illumination for simplicity. 


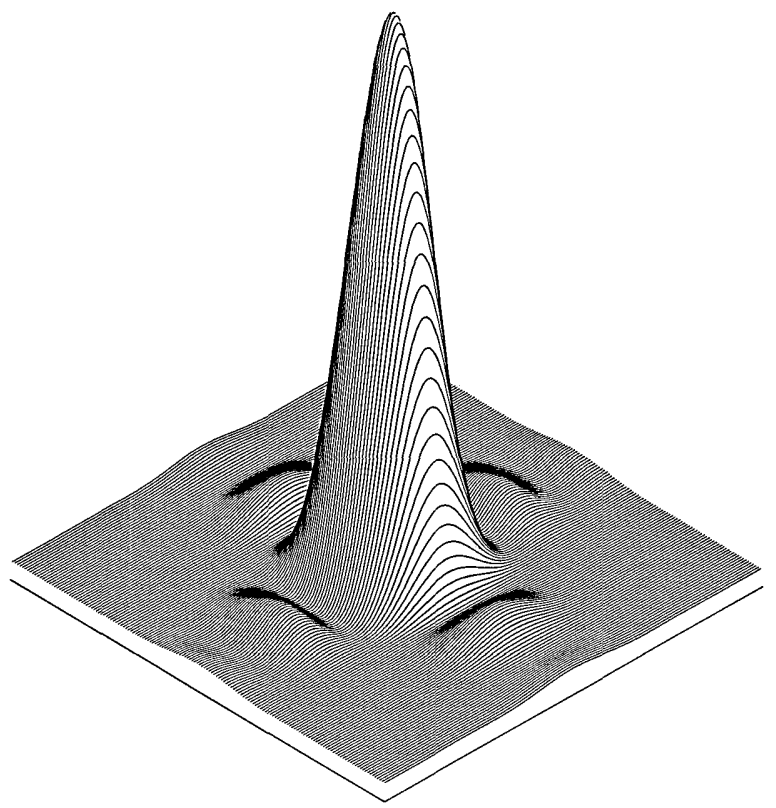

(a)

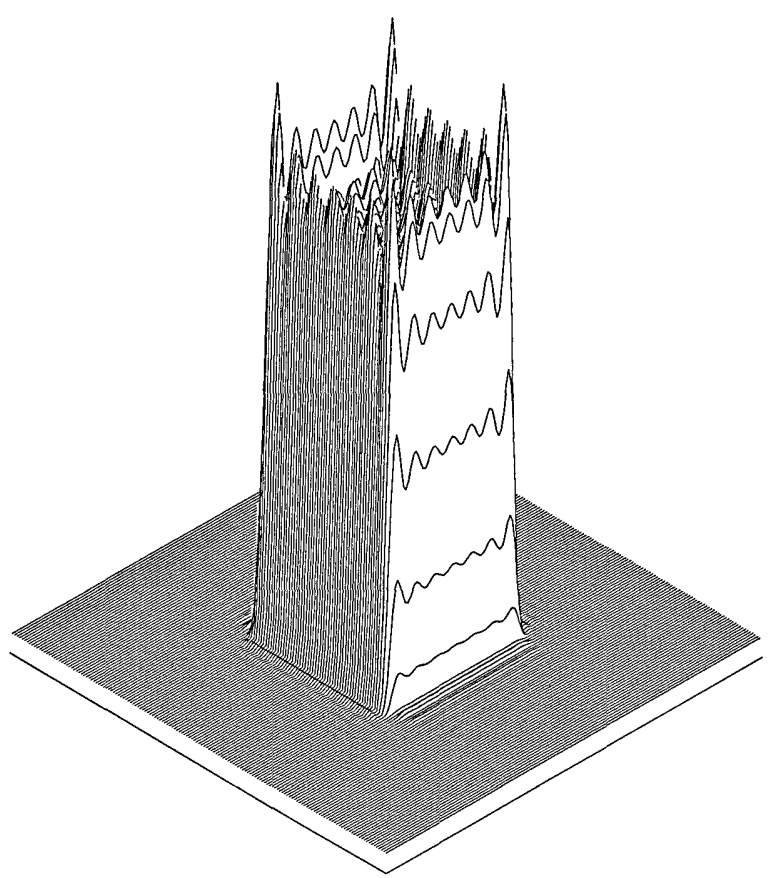

(b)

Fig. 3. Illustration of the asymptotic behavior of light-intensity distributions in the focal plane with $c_{x}=c_{y}=1$ and $\Delta x=\Delta y$ : (a) $W_{x}=W_{y}=0.53$, (b) $W_{x}=W_{y}=13.3$.

\section{Study of the Principal Focus}

We now study the evolution of the intensity in the image point $x_{i}=y_{i}=0$ of the $(0,0)$ order as the focal length encoded is changed. To this purpose, we rewrite Eq. (23), neglecting constant factors, as

$$
U_{0,0}(0,0)=f \int_{-\left(W_{x} c_{x}\right) / 2}^{\left(W_{x} c_{x}\right) / 2} \int_{-\left(W_{y} c_{y}\right) / 2}^{\left(W_{y} c_{y}\right) / 2} \operatorname{sinc}(u, v) \mathrm{d} u \mathrm{~d} v .
$$

We show that this function has an absolute maxi- mum with respect to the encoded focal $f$. First, if $f$ increases, the first factor obviously increases. With respect to the integral, the one-dimensional sinc integral reaches its maximum value when the integration interval is $(-1,1)$. This maximum value is approximately 1.17. If we consider different integration intevals (always symmetrical), the value of the integral is always lower. Starting from a small integration interval, the resulting value goes from 0 to 1.17. If the integration interval is increased outside $(-1,1)$, our result tends to 1 with oscillatory behavior. Then, if we start with a small value of $f$, $W_{x} c_{x} / 2 \gg 1$ and the integral takes a value near 1 . If $W_{x} c_{x} / 2$ decreases ( $f$ increases), Eq. (32) increases because $f$ increases. This is true for $\infty>W_{x} c_{x} / 2>$ 1 , as commented on above. For $W_{x} c_{x} / 2$ going from 1 to 0 , the integral decreases from its maximum value to zero. For very small intervals $\left(W_{x} c_{x} / 2 \rightarrow 0\right)$, a Taylor expansion of Eq. (32) leads easily to a $1 / f$ dependence. Then, $U_{0,0}(0,0)$ must have an absolute maximum versus $f$. This may be evaluated by numerical integration. For the special case in which $W_{x}=W_{y}$ and $c_{x}=c_{y}$, i.e., for a square device, the absolute maximum value is obtained for

$$
\frac{W_{x} c_{x}}{2}=0.685
$$

Using Eqs. (21) and (3) and using $L_{x}=N \Delta x$, we obtain the focal length that gives maximum intensity $\left|U_{0,0}(0,0)\right|^{2}$ :

$$
f_{\mathrm{opt}}=0.73 \frac{\Delta x \Delta x^{\prime} N}{\lambda} .
$$

Thus the LRFEL shows an important difference with respect to a Fresnel lenses encoded with infinite resolution: for an LRFEL there is a focal length giving maximum axial intensity. This is seen in Fig. 4. Here we show the intensity on the axis at the focal plane versus the encoded focal length when $N=$ $M, c_{x}=c_{y}=1$, and $\Delta x=\Delta y . \quad f_{\text {opt }}$ is defined by Eq. (34). There is a steep intensity decrease when the focal length is smaller than $f_{\text {opt }}$. The decrease is more gradual when the focal length encoded is greater than $f_{\text {opt. }}$. The evolution of the intensity distribution along the $x$ axis in the focal plane as a function of the focal length is shown in Figs. 4(b) and 4(c), seen from both sides with respect to $f$. Here the peak shape varies from a sinc to a rectangle.

The full width at half-maximum (FWHM) in the focal plane was also studied for the case $N=M, c_{x}=$ $c_{y}=1$, and $\Delta x=\Delta y$. The results are presented in Fig. 5(a). The dashed line represents the FWHM of the Fraunhofer diffraction pattern of the pupil. The width is normalized to the size $\Delta x^{\prime}$ of the pixels, and the focal length is divided by $f_{\text {opt. }}$. This graph illustrates several important results. First, for $f / f_{\text {opt }}<$ $\sim 1.5$, the width is smaller than the pixel size. Moreover, a minimum width is obtained near $f / f_{\text {opt }} \simeq$ 0.65 . Second, the asymptotic behavior already predicted is confirmed as $f / f_{\text {opt }} \rightarrow 0$ and $f / f_{\text {opt }} \rightarrow \infty$. 


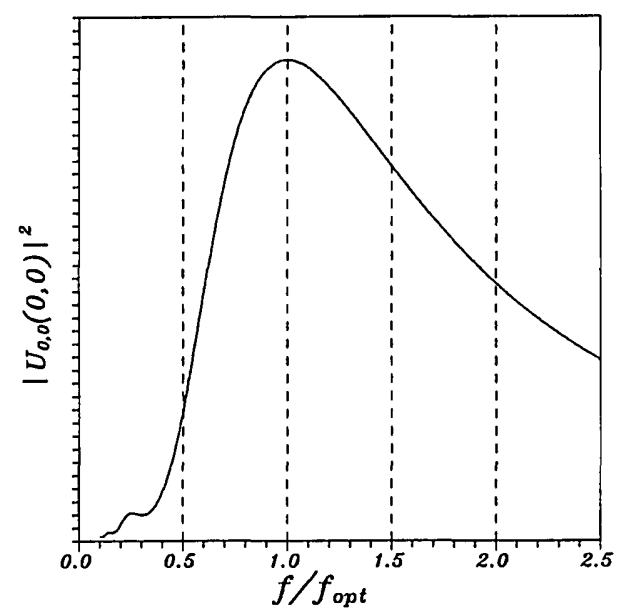

(a)

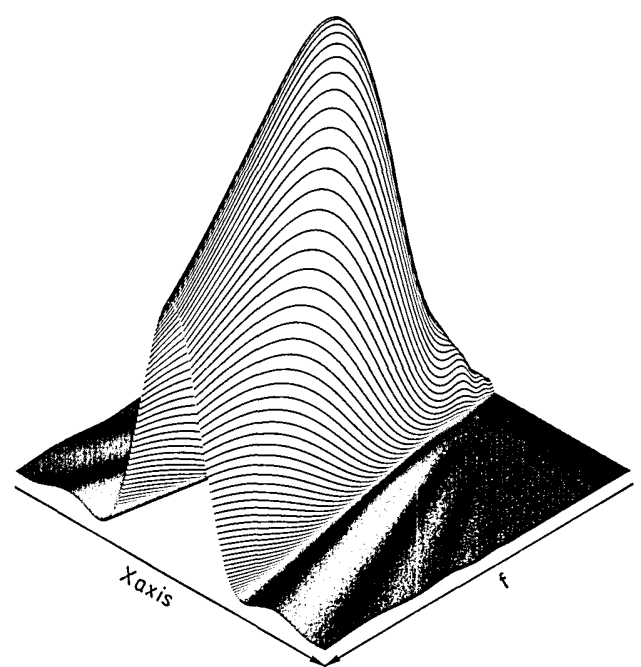

(b)

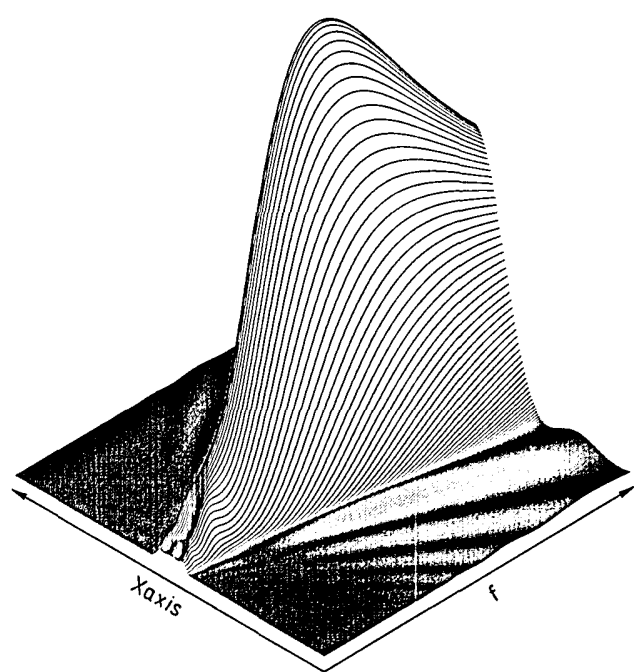

(c)

Fig. 4. Light intensity at the main focus versus the focal length encoded $(f)$. The focal length is normalized to $f_{\text {opt. }}$. The specific conditions are $c_{x}=c_{y}=1, \Delta x=\Delta y$, and $N=M$ : (a) evolution in the principal focus $\left(x_{i}=y_{i}=0\right)$, (b) evolution for $y_{i}=0$ on the $x$ axis for increasing $f$, (c) as in (b) for decreasing $f$.

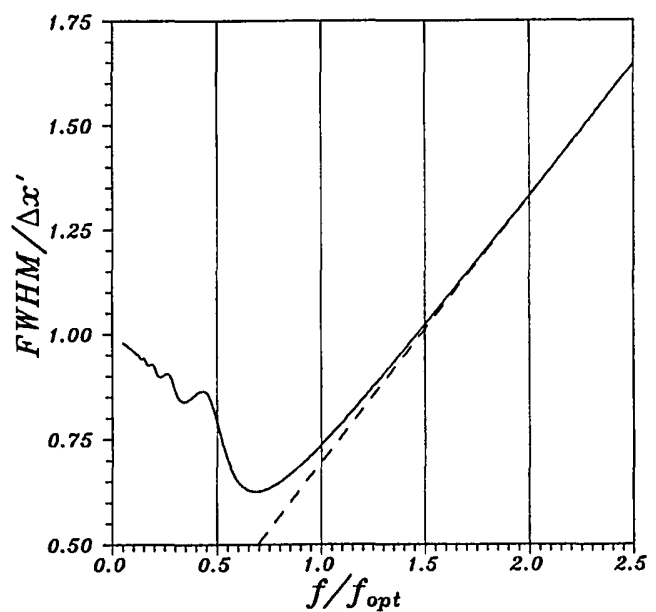

(a)

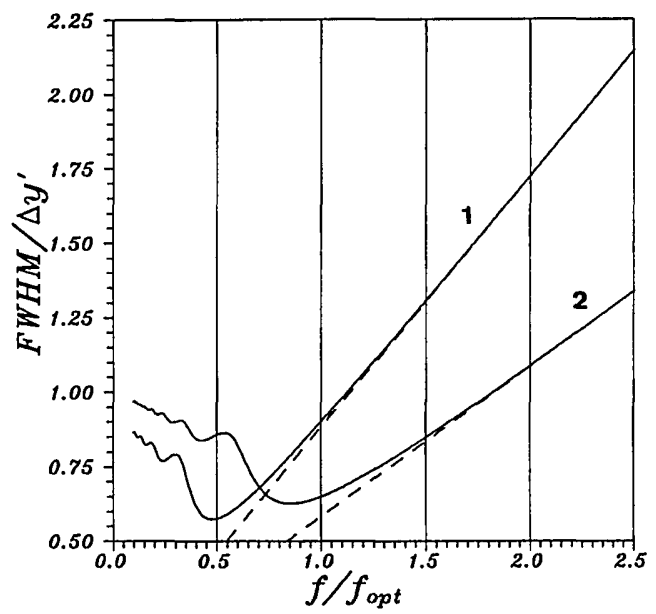

(b)

Fig. 5. FWHM of light-intensity distributions in the focal plane versus the focal length encoded. The focal length is normalized to $f_{\text {opt }}$ and the width is normalized to the size of the pixel: (a) $c_{x}=$ $c_{y}=1$ and $N=M$. (b) $c_{x}=0.69, c_{y}=0.67, N=320$, and $M=220$ (data corresponding to an Epson SLM ${ }^{13}$ ). Curve 1 corresponds to the width in the $x$ coordinate, and curve 2 corresponds to the $y$ coordinate.

Third, the width for $f=f_{\text {opt }}$ has an angular size, as seen from the diffracting device, which is slightly greater than the width of the peak originated by a lens encoded in infinite resolution (Fraunhofer diffraction pattern of the pupil, represented by the dashed line). Thus we can consider $f_{\text {opt }}$ as the optimum focal length to be encoded since, giving the maximum intensity at the principal focus, it also corresponds to a near-ideal FWHM. The appearance of the Fresnel encoded lens (binary version) corresponding to this focal length is shown in Fig. 6(a). Small fragments of secondary lenses can be seen [from Eq. (33) with $\left.W_{x}=1.37\right]$. If $c_{x}$ or $c_{y}$ is not 1 , secondary lenses may appear for $f=f_{\text {opt }}$, as shown Fig. 6(b). This uses the physical data of an Epson SLM. ${ }^{13}$ The corresponding curves of FWHM with respect to $f / f_{\text {opt }}$ are presented in Fig. 5(b). Of course, we now have two different curves for the two different lateral sizes.

An important feature of Eq. (34) is the presence of 


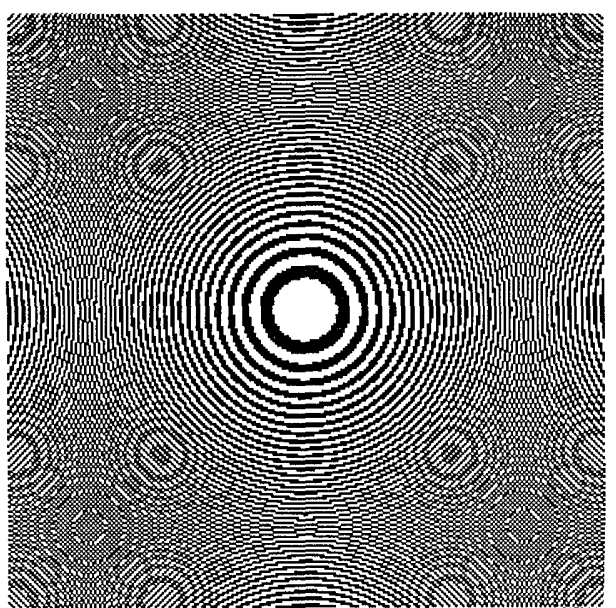

(a)

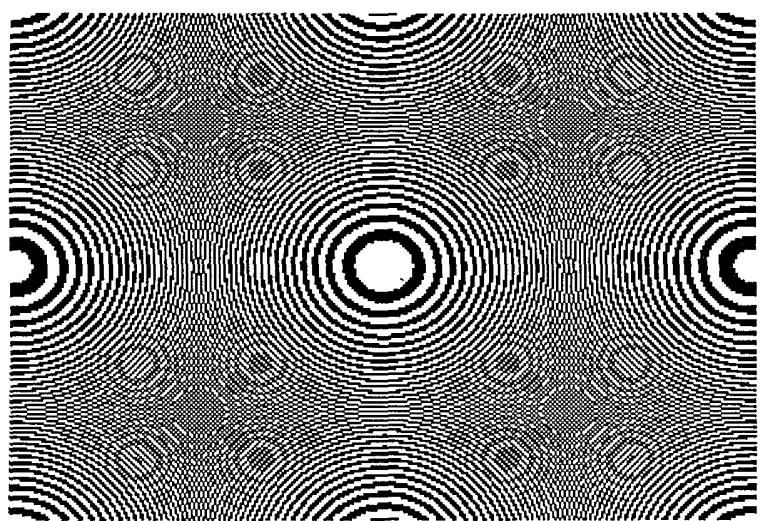

(b)

Fig. 6. Binary LRFEL corresponding to $f_{\text {opt }}$ : (a) $N=M=256$, $c_{x}=c_{y}=1$, and $\Delta x=\Delta y ;(b)$ data of an Epson SLM.

the factor $N$. This implies that too many pixels in the codification of a given $f$, which leads to the appearance of secondary-order lenses, diminishes the intensity in the main focal zone. In Section 5 we study the origin of this phenomenon and a means for correcting it.

\section{Optimization of the Codification of Short Focal-Length Lenses}

We have shown that when a relatively short focal length is being encoded, secondary lenses appear. This fact can be used to obtain arrays of lenses. ${ }^{10}$ This permits the use of LRFEL's as a multimaging system that replicates all the information reaching the device in the focal plane. However, if a single lens is to be encoded, the secondary lenses entail a loss of energy in the main focus and a degradation of image quality. ${ }^{3}$

If we need to use short focal distances, we can try to modify the codification procedure in order to concentrate as much light as possible at the principal focus. For this purpose we define blocks of pixels as a rectangle in the LRFEL of size $X / c_{x} \times Y / c_{y}$, centered at $\left[(n+1 / 2) X / c_{x},(m+1 / 2) Y / c_{y}\right]$, where $n$ and $m$ are arbitrary positive integers (including zero). We do not permit negative integers because the symmetry of the lens guarantees that all blocks can be represented by the block corresponding to positive $n$ and $m$. Then, a single $(n, m)$ pair represents four blocks.

We now calculate the contribution of each block $(n, m)$ to the principal focus. Within the previous assumptions, from Eq. (19) we can write

$$
\begin{aligned}
U_{0,0}^{n, m}(0,0)= & \int_{-1 / 2}^{1 / 2} \cos [2 \pi(n+1 / 2) u] \operatorname{sinc}(u) \mathrm{d} u \\
& \times \int_{-1 / 2}^{1 / 2} \cos [2 \pi(m+1 / 2) v] \operatorname{sinc}(v) \mathrm{d} v
\end{aligned}
$$

(constant phases are omitted for simplicity). The symmetry of the integration interval is taken into account. The final result is a product of two equivalent integrals that do not depend on specific details of the codification device. Each integrand is a cos modulated by a sinc and is always a real number. Thus each block always contributes to the focus as a real number that may be positive or negative.

Let us study the first integral, only in its positive range $(0,1 / 2)$ (the integrand is symmetric). In this interval the sinc function is always positive. When $n$ is even, the interval always contains $(n / 2)+1 / 4$ cycles of the cos, so the integral is positive. If $n$ is odd, the interval always contains $(n-1) / 2+3 / 4$ cycles, and it is negative. The sign of Eq. (35) is then given by

$$
\operatorname{sign}=(-1)^{n+m} .
$$

As the order of the block [defined by $(n, m)$ ] increases, its contribution to the principal focus decreases because the interval contains more and more oscillations of the cos function in Eq. (35). If the four central blocks $(0,0)$ of an LRFEL are fully encoded in the diffracting device, their contributions are positive. The four blocks always define an area at least 4 times greater than the apparent area associated with only one lens given by $X \times Y$. For a general case, $c_{x}$ and $c_{y}$ are less than unity, so these four blocks contain the $(0,0)$ lens and secondary lenses. If we add the twelve neighboring blocks in the codification, eight of them contribute with a negative sign and four with a positive sign, and the result is a loss of intensity.

Table 1. Values of the First Integral of Eq. (35) for Several $n$

\begin{tabular}{lc}
\hline$n$ & Normalized Value $^{a}$ \\
\hline 0 & 1.0000 \\
1 & -0.2346 \\
2 & 0.1383 \\
3 & -0.0990 \\
4 & 0.0763 \\
5 & -0.0630 \\
6 & 0.0526 \\
7 & -0.0462 \\
8 & 0.0401 \\
9 & -0.0365 \\
\hline
\end{tabular}

${ }^{a}$ The integral is normalized to the case of $n=0$. 
Table 2. Intensity in the Focus for the Optimized and Nonoptimized Lenses ${ }^{a}$

\begin{tabular}{ccc}
\hline $\begin{array}{c}\text { Number of } \\
\text { Blocks }\end{array}$ & Optimized & $\begin{array}{c}\text { Not } \\
\text { Optimized }\end{array}$ \\
\hline $2 \times 2$ & 1.000 & 1.000 \\
$4 \times 4$ & 2.324 & 0.343 \\
$6 \times 6$ & 3.552 & 0.666 \\
$8 \times 8$ & 4.693 & 0.419 \\
\hline
\end{tabular}

aThe intensity is normalized to the case in which only $2 \times 2$ blocks are encoded.

This is illustrated in Table 1 and the third column of Table 2.

From this latter result, we can devise a simple lens optimization procedure: we shift the phase of the blocks that contribute with negative sign in $\pi$. For

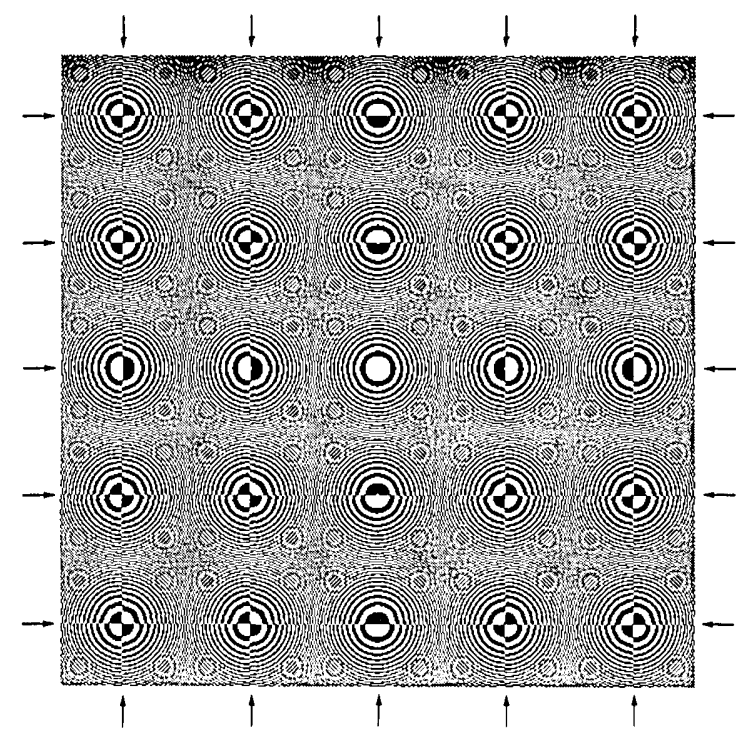

(a)

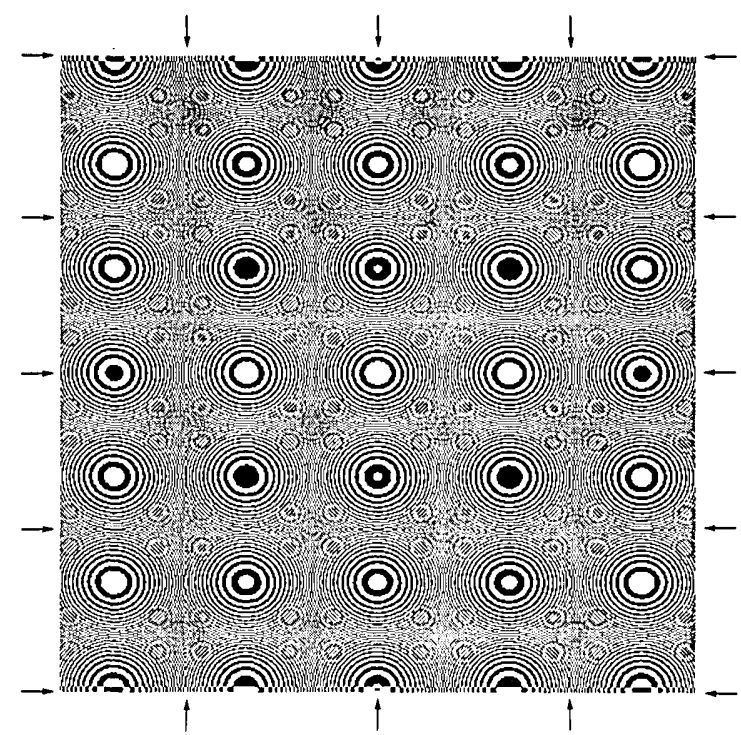

(b)

Fig. 7. Binary LRFEL optimized for: (a) $c_{x}=c_{y}=1, \Delta x=\Delta y$, and $W_{x}=W_{y}=5$; (b) the data of an Epson SLM, but $N=M=$ 480. The lines dividing blocks are indicated by arrows. low-resolution Fresnel zone plates this is done simply by a reversal of the contrast of the pixels. ${ }^{14,15}$ If we use this method for $c_{x}=c_{y}=1$, figures such as Fig. 7(a) are obtained with binary coding. The four blocks that define a secondary lens have opposite signs, and their contribution to the secondary focus is exactly zero. ${ }^{14,15}$ Small contributions to these secondary foci arise from the other blocks. An optimized lens for the Epson SLM is shown in Fig. 7(b). Here blocks are greater because $c_{x}=0.69$ and $c_{y}=0.67$, i.e., less than 1. Nevertheless, blocks of the same order in Fig. 7 always make the same contribution to the principal focus. Then, if $c_{x}$ and $c_{y}$ are different than 1 , to obtain the same contribution as for $c_{x}=c_{y}=1$, we must increase the area by a factor $1 /\left(c_{x} c_{y}\right)$. From Table 2 we can conclude that the optimization method works as expected.

In Fig. 8 we show a comparison of the axial intensities at the focus for the optimized and the nonoptimized lens versus the focal length, for $c_{x}=$ $c_{y}=1$. The two intensities coincide when the size of the LRPEL is smaller than $2 \times 2$ blocks. The optimized lens permits the codification of short focal lengths without significant loss of energy.

In Fig. (9) the normalized FWHM of the lightintensity distribution in the main focus is represented versus $f$. The dashed line indicates the width for a lens of infinite resolution. Curve 1 corresponds to the optimized lens and curve 2 corresponds to the nonoptimized. It is remarkable that the optimized lens keeps a width similar to that of the infiniteresolution case. Thus an optimized lens can give very narrow peaks with respect to the size of the pixel. In Fig. (10) we show the light distribution on the $x$ axis for increasing focal lengths in the zone of short $f$. The light distribution is always a narrow peak and does not tend to a rectangular shape, as in Fig. 4(c).

Furthermore, the proposed optimization method is the one that gives the highest intensity in the center of the principal focus. To demonstrate this, we consider the contribution of any rectangular pixel at

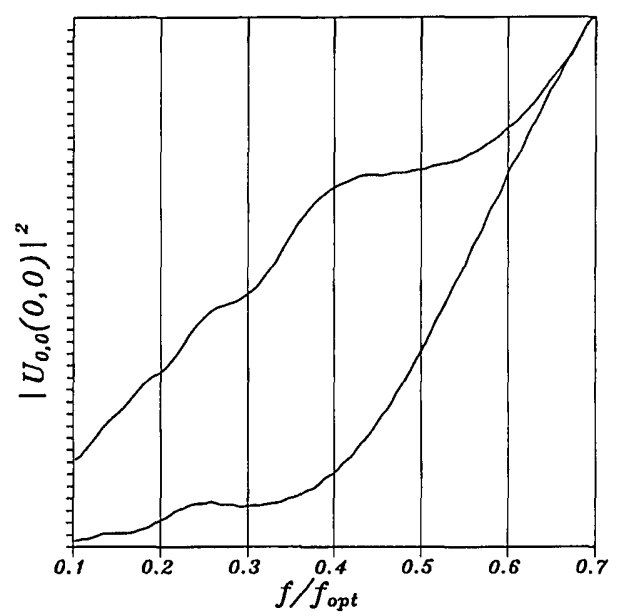

Fig. 8. Comparison of light intensities at $x_{i}=y_{i}=0$ for a LRFEL (lower curve) and for an optimized LRFEL (upper curve) $\left(c_{x}=c_{y}=\right.$ $1, N=M$, and $\Delta x=\Delta y$ ). 'I'he latter always gives more energy. 


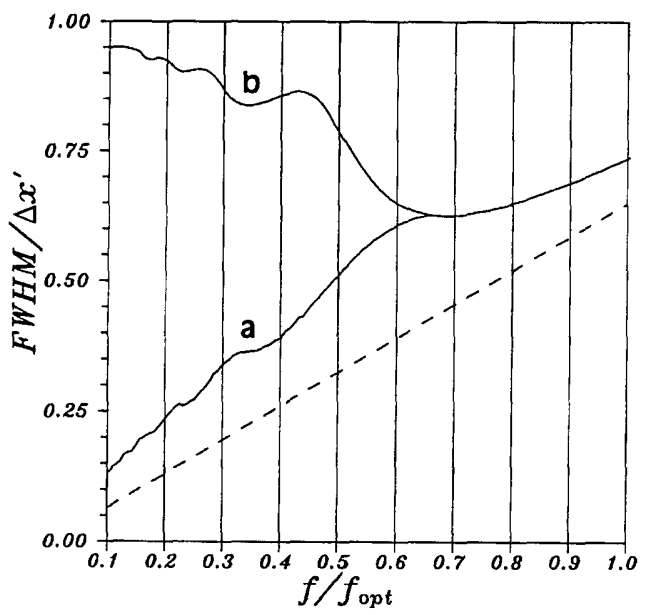

Fig. 9. Comparison of FWHM of light distributions for (a) the optimized and (b) the nonoptimized lens. The dashed line corresponds to the Fraunhofer diffraction of the pupil.

the $(0,0)$ focal region in terms of our parameters:

$$
\begin{aligned}
\frac{\lambda f}{\left(X / c_{x}\right)\left(Y / c_{y}\right)}\left(A\left(x_{n}, y_{m}\right)\right. & \left.\exp \left\{\frac{i k}{2 f}\left[\left(x_{i}-x_{n}\right)^{2}+\left(y_{i}-y_{m}\right)^{2}\right]\right\}\right) \\
& \times \operatorname{sinc}\left(\frac{x_{i}-x_{n}}{\left(X / c_{x}\right)}, \frac{y_{i}-y_{m}}{\left(Y / c_{y}\right)}\right),
\end{aligned}
$$

where $x_{n}$ and $y_{m}$ represent the shift of the rectangle from the $(0,0)$ focus [Eq. (2)]. This expression is derived with the same assumptions as in section 3 of Ref. 10. This expression can be interpreted as having two terms. The first, the complex exponential, is the contribution of a source point centered at $\left(x_{n}, y_{m}\right)$.

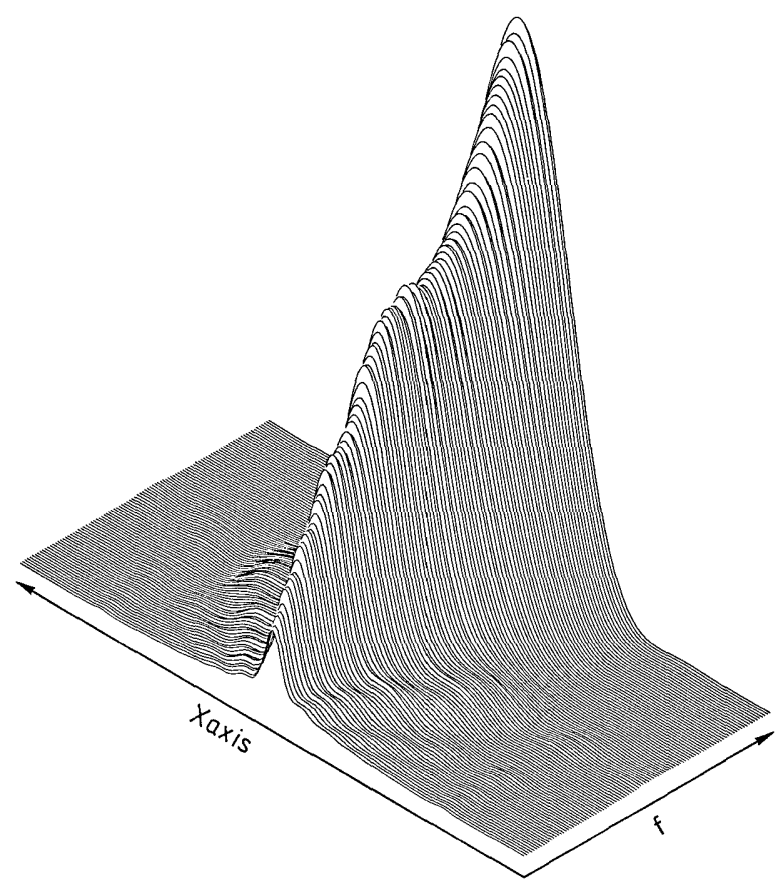

Fig. 10. Evolution of light-intensity distributions on the $x$ axis for increasing focal length for the optimized lens.
The second, the sinc, can be interpreted as a correction because of the finite extension of the pixel. This comment is valid for any pixel shape. For $x_{i}=y_{i}=0$ we have

$$
\begin{aligned}
\frac{\lambda f}{\left(X / c_{x}\right)\left(Y / c_{y}\right)}\left\{A\left(x_{n}, y_{m}\right)\right. & \left.\exp \left[\frac{i k}{2 f}\left(x_{n}^{2}+y_{m}^{2}\right)\right]\right\} \\
& \times \operatorname{sinc}\left(\frac{x_{n}}{\left(X / c_{x}\right)}, \frac{y_{m}}{\left(Y / c_{y}\right)}\right) .
\end{aligned}
$$

The first term implies that if a device consists of $N \times$ $M$ perfect points, an LRFEL can be encoded as in Section 1. In this ideal case, if $A\left(x_{n}, y_{m}\right)$ is a Fresnel phase-encoded lens, the complex exponential term vanishes, and all points of the device contribute to the amplitude in the focus with the same modulus and with the same phase angle; as a consequence there is no negative interference. This can also be observed if we note that, when $c_{x}$ and $c_{y}$ tend to zero, the size of the first four blocks $(0,0)$ tends to be infinite.

When pixels are finite and particularly when they are rectangular, for the point $x_{i}=y_{i}=0$ the LRFEL can be seen as $N \times M$ perfect points whose amplitude contribution is modified by the sinc function. The sinc in expression (38) is not always positive; it has periodical changes of sign with a periodicity given by $X / c_{x}$ and $Y / c_{y}$, which coincides with the size and the location of blocks. This fact justifies the optimization method. Thus if we codify an optimized Fresnel phase-encoded lens, the contribution of each pixel always has the same phase angle, and we obtain the maximum contribution allowable. When a pixel lies between two blocks, Eq. (38) implies that it must be included where its center belongs. If it is on the border, its contribution is null, as seen from the same equation.

Important conclusions may be drawn from these facts. First, if pixels were perfect points, when an LRFEL is encoded, all pixels would have an optimum contribution at the focus, with a phase given by Eq. (7). Second, because of the finite extension of the pixel, for each focus the lens can be considered as $N \times$ $M$ perfect points multiplied by the Fourier transform of the pixel, centered at the focus coordinates. This implies that several groups of pixels can contribute with inadequate phase to the focus because of the change of sign (or in general, the changing phase) of the FT. In our case the pixel was a small rectangle, and its FT was the sinc. This function changes sign periodically, making groups of pixels that contribute negatively. We call these groups of pixels blocks. Then, a block can be interpreted as a group of pixels that contributes with the same sign to the focus. For the special case of rectangular pixels the relative contribution of the different blocks does not depend on the specific properties on the device. The contribution of a block decays with its order because the sinc function also decays. If the device is a phase modulator, we can directly assign the phase of the quadratic exponential and add $\pi$ when codifying in an opposite block. 


\section{Summary}

In this paper we have described the effects of the codification of Fresnel lenses in low-resolution devices and their performances in the focal plane. The main results are the following:

(i) When a single Fresnel lens is encoded in a low-resolution device, new secondary lenses may appear, giving rise to an array of lenses.

(ii) Each secondary lens has a different phase, depending on the resolution of the device and the focal length encoded.

(iii) Each secondary lens has an apparent size, but it has been demonstrated that it behaves as if encoded in all the device.

(iv) The complex amplitude originated by the propagation of any distribution encoded in a lowresolution device (for instance, a Fresnel lens) can be calculated: first we suppose that the pixels are perfect points (mathematically, Dirac dela functions), and eventually we convolve with the function that defines the transmittance of a single pixel.

(v) For each focal region the light distribution can be calculated as the convolution of the Fourier transform of the pupil (as seen from each focus) with the function that defines the shape of the pixel.

(vi) When many secondary lenses appear at the device because of its low resolution, the intensities in the corresponding focal regions are similar. Moreover, the intensity, corresponding to the secondary lenses that do not appear because of the finite size of the pupil, tends to be zero. When we have only a single lens, the intensity of the central point of each focus is modulated by a sinc function. For the particular case in which the center-to-center distance between the pixels coincides with the pixel width, the zeros of the sinc coincides with the central points.

(vii) For short focal lengths the shape of the light distribution at any focus tends to the shape of the pixel. For long focal distances the shape tends to be the Fourier transform of the pupil function.

(viii) There is a focal length that gives maximum intensity at the central point of the principal focus. The light distribution corresponding to this maximum has a FWHM that is comparable with the width obtained by use of an infinite-resolution lens bounded by the same pupil. This fact permits us to define this focal length as the optimum focal length for the principal focus.

(ix) For any principal or secondary lens encoded the contribution of each pixel to the central point of each focus can be understood as the product of a spherical wave emerging from the center of the pixel and the Fourier transform of the function that defines the shape of the pixel. For a rectangular pixel the Fourier transform is a sinc that changes sign periodically. This implies that certain groups of pixels contribute with a $\pi$ phase shift, diminishing the intensity in the focal region. A group of pixels contributing with the same sign has been defined as a block.

(x) For rectangular pixels the contribution of a block does not depend on the details of the lowresolution device.

Finally, this theory describes the focusing properties of low-resolution Fresnel-encoded lenses and permits the optimization for a single short focallength lens codification. Thus this paper may be a starting point for further studies on practical uses of low-resolution lenses.

This study was supported in part by the Comisión Interministerial de Ciencia y Tecnologia (project ROB91-0554).

\section{References}

1. F. T. S. Yu, X. Yang, and D. A. Gregory, "Polychromatic neural networks," Opt. Commun. 88, 81-86 (1992).

2. A. Bergeron, G. V. April, and H. H. Arsenault, "Recording holograms with diagonal coding on binary spatial light modulators for pattern recognition," Appl. Opt. 29, 1652-1657 (1990).

3. E. C. Tam, S. Zhou, and M. R. Feldman, "Spatial-lightmodulator-based electro-optical imaging system," Appl. Opt. 31, 578-580 (1992).

4. J. A. Davis, H. M. Schley-Seebold, and D. M. Cottrell, "Anamorphic optical systems using programmable spatial light modulators," Appl. Opt. 31, 6185-6186 (1992).

5. J. A. Davis, S. H. Drayton, D. M. Cottrell, and J. E. Davis, "Improved synthetic discriminant function performance using Fresnel lens-encoded binary phase-only filters," Appl. Opt. 29, 2594-2599 (1990).

6. J. L. Horner and J. R. Leger, "Pattern recognition with binary phase-only filters," Appl. Opt. 24, 609-611 (1985).

7. J. A. Davis, D. M. Cottrell, J. E. Davis, and R. A. Lilly, "Fresnel lens-encoded binary phase-only filters for optical pattern recognition," Opt. Lett. 14, 659-661 (1989).

8. J. A. Davis, D. M. Cottrell, R. A. Lilly, and S. W. Connely, "Multiplexed phase-encoded lenses written on spatial light modulators," Opt. Lett. 14, 420-422 (1989).

9. O. Bryngdahl, "Moiré: formation and interpretation," J. Opt. Soc. Am. 64, 1287-1294 (1974).

10. D. M. Cottrell, J. A. Davis, T. R. Hedman, and R. A. Lilly, "Multiple imaging phase-encoded optical elements written as programmable spatial light modulators," Appl. Opt. 29, 25052509 (1990).

11. J. W. Goodman, Introduction a l'Optique de Fourier et a l'Holographie (Masson, Paris, 1972).

12. J. D. Gaskill, Linear Systems, Fourier Transforms, and Optics (Wiley, New York, 1978).

13. J. C. Kirsch, D. A. Gregory, M. W. Thie, and B. K. Jones, "Modulation characteristics of the Epson liquid-crystal television," Opt. Eng. 31, 963-970 (1992).

14. S. B. Viñas, Z. Jaroszewicz, A. Kolodziejczyk, and M. Sypek, "Zone plates with black focal spots," Appl. Opt. 31, 192-198 (1992).

15. J. Ojeda-Castañeda, P. Andrés, and M. Martínez-Corral, "Zero axial irradiance by annular screens with angular variation," Appl. Opt. 31, 4600-4602 (1992). 